\title{
FORUM
}

\section{Das Mandat für die Regierungskonferenz - Die zweite Chance}

\author{
Andrew Duff*
}

Ermattet ging der Europäische Rat am Samstag, den 23. Juni 2007, um 4:35 Uhr, zu Ende. Eine einzuberufende Regierungskonferenz wurde mit der Aufgabe betraut, so viel wie möglich vom Verfassungsvertrag aus dem Jahr 2004 zu retten.

Obwohl die Grundzüge der Vertragsänderung klar sind und das Mandat strikter und präziser als üblich formuliert wurde, wird die Regierungskonferenz die schwierige Aufgabe haben, politische Unsicherheiten zu beseitigen, Vereinbarungen abzuklären und zu bekräftigen sowie rechtliche Kohärenz sicherzustellen. Eine erfolgreiche Regierungskonferenz würde zudem die europäischen Institutionen und die Mitgliedstaaten darauf vorbereiten, den endgültigen Vertrag rechtzeitig vor den Wahlen des Europäischen Parlaments im Juni 2009 zu ratifizieren.

In Anerkennung der wachsenden konstitutionellen Befugnis des Europäischen Parlaments, werden drei Repräsentanten (keine Beobachter) des Europäischen Parlaments an der Regierungskonferenz teilnehmen. Das Europäische Parlament wurde zudem ersucht, bis Oktober einen Vorschlag zur Neuverteilung der Sitze vorzulegen. Die Verteilung der 736 Sitze im Europäischen Parlament ist für die Periode 2009-2014 durch die 20. Erklärung des Nizza-Vertrags vorab festgelegt. Der Verfassungsvertrag erhöht diese Anzahl jedoch auf 750, wobei die Deutschen mit 96 Abgeordneten drei Abgeordnete weniger und die Malteser mit sechs Abgeordneten einen mehr stellen werden. Das entsprechende Protokoll 34 des Verfassungsvertrags wird nun neu zu verhandeln sein, damit es entweder ab 2009 oder ab 2014 in Kraft treten kann.

\section{,Verfassung plus 6}

Als Erfolg des Europäischen Rates sind diejenigen Entscheidungen zu betrachten, die der Regierungskonferenz das Mandat erteilen, neue Bestimmungen zur Sicherung der Energieversorgung und zur Förderung der Zusammenlegung der Energienetze sowie zur Notwendigkeit einer Energiepolitik ,,im Geiste der Solidarität zwischen den Mitgliedstaaten“ aufzunehmen. Dies ist von besonderer Bedeutung für Polen. Willkommen und notwendig war die Vereinbarung, die Bekämpfung des Klimawandels zu den Zielen der Umweltpolitik hinzuzufügen. Ein nützlicher neuer Artikel, der die Rolle der nationalen Parlamente kodifiziert, wird eingefügt werden. Außerdem wird es einen Verweis auf die Kopenhagener Kriterien und andere Beitrittskriterien geben, die vom Europäischen Rat festgelegt wurden beziehungsweise werden. Dies hat den wichtigen Effekt, dass die Beitrittskriterien und Prozesse justiziabel werden - für die Beitrittskandidaten und auch für die Mitgliedstaaten.

Man kann sagen, dass all diese Änderungen auf viele der Befürchtungen in der Öffentlichkeit eingehen, die insbesondere während der Referendumkampagnen in Frankreich und den Niederlanden geäußert worden sind. Zudem wird der Tatsache Rechnung getragen, dass Klimawandel und Energiethemen seit dem ursprünglichen Verfassungsvertrag von 2004 an Bedeutung gewonnen haben.

* Andrew Duff, MdEP (Vereinigtes Königreich, Fraktion der Allianz der Liberalen und Demokraten für Europa, ALDE), ist einer der drei Repräsentanten des Europäischen Parlaments, die an der Regierungskonferenz 2007 teilnehmen. 


\section{Kompliziertheit}

Die Suche nach einem „Mini-“ oder vereinfachten Vertrag wurde aufgegeben. Der Europäische Rat beschloss, den Verfassungsvertrag von 2004 komplett zu restrukturieren. Ein Reformvertrag wird die bestehenden Verträge ändern, aber nicht ersetzen: nämlich erstens, den Vertrag über die Europäische Union (vergleichbar mit den Teilen I und IV des Verfassungsvertrags) und zweitens, den Vertrag über die Arbeitsweise der Union (vorher Vertrag zur Gründung der Europäischen Gemeinschaft, vergleichbar mit Teil III des Verfassungsvertrags). Beide geänderten Verträge werden den gleichen rechtlichen Status haben und gleichzeitig (oder nie) in Kraft treten.

Die Union wird eine einheitliche Rechtspersönlichkeit erhalten und der Europäischen Konvention zum Schutz der Menschenrechte und Grundfreiheiten beitreten. Die , dritte Säule' der EU - die Justiz- und Innenpolitik -, die in Maastricht (1992) begründet wurde, wird völlig von der Gemeinschaftsmethode (die ,erste Säule‘) umfasst werden. Der Vorrang des EU-Rechts aus Artikel I-6 des Verfassungsvertrags wird zwar nicht aufgegriffen, aber die gängige Rechtsprechung und -auffassung in dieser Sache wird durch eine Erklärung bestätigt.

Von großer Wichtigkeit ist, dass die meisten der Änderungen bezüglich der Haushalts-, Gesetzgebungs- und Entscheidungsverfahren der Union, die im Verfassungsvertrag von 2004 vereinbart wurden, in einen der beiden geänderten Verträge eingearbeitet werden. Die Präambel des Verfassungsvertrags wurde weitgehend abgeschafft, ebenso wie der größte Teil des Artikels I-1 zu den Prinzipien, die die Union begründen sowie der Artikel I-8 zu den Symbolen der Union.

\section{Die Charta der Grundrechte}

Die Charta der Grundrechte (Teil II des Verfassungsvertrags) wird nunmehr als Anhang der Verträge veröffentlicht werden. Die Charta wird jedoch einen Querverweis in dem geänderten EU-Vertrag erhalten, der ihr, unter Vorbehalt der vereinbarten Ausnahmen ihres Anwendungsbereichs, dieselbe Rechtsverbindlichkeit wie den Verträgen zuspricht. Das Vereinigte Königreich bekommt allerdings ein Protokoll, das versucht, die Wirkung der Charta zu verringern, indem es den Europäischen Gerichtshof und die nationalen Gerichten daran hindert, die Charta in Bezug auf die Praktiken oder das Handeln des Vereinigten Königreichs als geltendes einklagbares Recht anzusehen. Vorbehalte gibt es insbesondere gegenüber der Ausdehnung von sozialen Rechten. Dies sind schlechte Neuigkeiten für britische Bürger und EU-Bürger die im Vereinigten Königreich leben.

Das Protokoll scheint zudem rechtlich fehlerhaft. ${ }^{1}$ Trotz der britischen Ausschluss-Regel werden die EU Gerichte nicht umhin kommen, Rechtsprechung in Grundrechtsfragen zu entwickeln, die sich zu generellen Prinzipien des EU-Rechts entwickeln werden, an die sich alle Mitgliedstaaten halten müssen. Zudem wird für den Europäischen Gerichtshof die Nationalität eines EU-Bürgers, der sich auf die Charta in der Zuständigkeit der Europäischen Union beruft, keine Rolle spielen.

Polen bekommt eine einseitige Erklärung, die versucht zu verhindern, dass die Charta verwendet wird, um die nationale Gesetzgebung in den Bereichen der öffentlichen Sittlichkeit oder des Familienrechts zu beeinflussen. Polen hat zudem Interesse geäußert, es dem Vereinigten Königreich gleichzutun und zu versuchen, ein opt-out aus der Charta zu erhalten. Dies führte zu Befürchtungen vor einem euroskeptischen spill-over.

1 Diese Lösung ist, obschon holperig, dem ersten Vorschlag des Vereinigten Königreiches vorzuziehen, welcher den Anwendungsbereich der Charta auf die EU-Institutionen begrenzen wollte und nicht auf die Mitgliedstaaten. 


\section{Außenpolitik}

Der im Verfassungsvertrag vorgeschlagene Außenminister der Union wird zwar seine Funktionen und Kompetenzen, einschließlich des Auswärtigen Dienstes, behalten, verliert jedoch seinen Namen. Er wird nun zum HVUASP „Hohen Vertreter der Union für Außenund Sicherheitspolitik".

Die Bestimmungen des Verfassungsvertrags über die Gemeinsame Außen- und Sicherheitspolitik, einschließlich derer zur ständigen strukturierten Zusammenarbeit im Verteidigungsbereich, werden im Wesentlichen ohne Änderungen übernommen. Als Zugeständnis an das Vereinigte Königreich wird jedoch eine Erklärung ergänzt werden, die eine minimale Interpretation der Verpflichtungen der Gemeinsamen Außen- und Sicherheitspolitik gibt, unter anderem um zu versuchen, den ständigen Sitz des Vereinigten Königreichs im Sicherheitsrat der Vereinten Nationen zu schützen. Auf britischen Wunsch wird zudem eine besondere Rechtsgrundlage für den Datenschutz im GASP-Bereich entwickelt werden.

\section{Terminologie}

Abgesehen vom neu eingeführten Begriff HVUASP sind einige terminologische Änderungen notwendig, so wurden die Begriffe ,Verfassung' und ,Gemeinschaft" abgeschafft. Auch auf ,Gesetz' und ,Rahmengesetz' wird zugunsten der bestehenden ,Verordnungen', ,Richtlinien“ und ,Beschlüsse‘ verzichtet. Die Hierarchie der Instrumente wird entsprechend anzupassen sein - jedoch vorsichtig, damit der Einflussgewinn des Parlaments, der 2004 mit dem Verfassungsvertrag erzielt werden konnte, hinsichtlich der Parität von Parlament und Rat bei der Wahl der Instrumente, der Übertragung von Kompetenzen an die Kommission und deren Kontrolle erhalten bleibt.

\section{Nationale Parlamente}

Auf Antrag der Niederlande und Frankreichs wird der Zeitraum, der den nationalen Parlamenten zur Verfügung steht, um mit einem Drittel der nationalen Parlamente Entwürfe für Gesetzgebungstexte zu prüfen und begründete Stellungnahmen die Subsidiarität betreffend vorzulegen, von sechs auf acht Wochen verlängert. Dieser Frühwarnmechanismus - die Gelbe Karte - wird durch eine neues Verfahren ergänzt. Wenn die Kommission einen Gesetzesvorschlag auch bei der Anfechtung von mehr als der Hälfte der nationalen Parlamente beibehalten will, wird ihre begründete Stellungnahme zusammen mit der begründeten Stellungnahme der Parlamente an die EU-Gesetzgeber (das Europäische Parlament und den Rat) übermittelt. Diese prüfen dann, ob der Gesetzesvorschlag mit dem Subsidiaritätsprinzip im Einklang steht. Bei diesem neuen Verfahren, das man als ,Orange Karte ' bezeichnen könnte, handelt der Rat mit der Mehrheit von 55 Prozent seiner Mitglieder und das Parlament mit der Mehrheit der abgegeben Stimmen - ob jedoch die beiden ,Kammern' einzeln oder gemeinsam handeln müssen ist einer der Punkte, die während der Regierungskonferenz entschieden werden müssen. In jedem Fall ist jedoch die Tatsache, dass sich die nationalen Parlamente weniger an die Kommission und stärker an die Gesetzgeber wenden müssen, sehr willkommen zu heißen.

Als Entgegenkommen an die Euroskeptiker bekommt jedes nationale Parlament ein Vetorecht gegen europäische Gesetzgebung im Bereich des Familienrechts.

\section{Abstimmungen im Rat}

Als Reaktion auf die polnische Forderung, die Quadratwurzel für die Stimmenberechnung im Rat anzuwenden, werden nun die folgenden Regelungen vorgesehen: 
- Bis 2014: Das Beschlussverfahren mit qualifizierter Mehrheit gemäß den Nizza-Regelungen plus Ioannina-Mechanismus. Dieser wurde 1994 eingeführt, um eine Fortführung der Verhandlungen zu erlauben, wenn Mitglieder des Rates, die mindestens drei Viertel der Bevölkerung oder mindestens drei Viertel der Mitgliedstaaten repräsentieren, Einspruch gegen die Annahme eines Rechtsaktes mit qualifizierter Mehrheit einlegen. ${ }^{2}$

- Zwischen 2014-2017: Das Verfahren der Beschlussfassung mit doppelter Mehrheit gemäß dem Verfassungsvertrag (das heißt, 55 Prozent der Mitglieder des Rates, die 65 Prozent der Bevölkerung repräsentieren) plus der Möglichkeit, die Anwendung der Nizza-Regelungen und des Ioannina-Mechanismus zu beantragen.

- Ab 2017: Das Verfahren der Beschlussfassung mit doppelter Mehrheit plus Ioannina-Mechanismus.

Der Rat wird diese ungewollte Verzögerung und Vernebelung bei der Einführung seines neuen Verfahrens der Beschlussfassung noch bedauern. Es sei daran erinnert, dass der Verfassungsvertrag ursprünglich am 1. November 2006 in Kraft treten sollte.

Sogar noch vor dem offiziellen Beginn der Regierungskonferenz am 23. Juli, stellte die polnische Regierung das Mandat hinsichtlich des Ioannina-Mechanismus in Frage. Warschau behauptete, dass vereinbart worden wäre, dass die Anwendung von ,Ioannina“ jeden legislativen Fortgang für zwei Jahre blockieren würde. Alle anderen glaubten, dass es nur zu einer Verzögerung bis zum nächsten Treffen des Europäischen Rates - in anderen Worten, maximal vier bis fünf Wochen - kommen würde.

\section{Kompetenzen}

Die Tschechische Republik, die Niederlande, Polen und das Vereinigte Königreich setzten eine Erklärung durch, die Zuständigkeiten strikter abzugrenzen. Dies bedeutet, dass eine zukünftige Regierungskonferenz sowohl Zuständigkeiten zurück an die Mitgliedstaaten als auch neue Zuständigkeiten an die Union übertragen kann.

Es wird zudem ein Protokoll geben, um die vermeintliche Ausbreitung der Kompetenz der EU, in Bereichen mit geteilter Zuständigkeit von EU und Mitgliedstaaten, jenseits des spezifischen Bereichs der bereits bestehenden Gesetzgebung zu stoppen.

Es wird explizit festgehalten werden, dass die nationale Sicherheit in der alleinigen $\mathrm{Zu}-$ ständigkeit der einzelnen Mitgliedstaaten verbleibt. Eine neue Bestimmung wird eingefügt werden, um sicherzustellen, dass die nationale Sicherheit im Bereich der Verwaltungszusammenarbeit Vorrang vor EU-Verpflichtungen hat.

Als Zugeständnis an die Niederlande und die Tschechische Republik wird die sogenannte Flexibilitätsklausel um Erklärungen ergänzt werden, die sicherstellen sollen, dass die Anwendung der Flexibilitätsklausel die Kompetenzen der Union nicht unmäßig weit ausdehnt, insbesondere nicht im Bereich der Außenpolitik. ${ }^{3}$

Als Zugeständnis an das Vereinigte Königreich wurde im Verfassungsvertrag bezüglich der Bestimmungen auf dem Gebiet der sozialen Sicherheit zur Herstellung von Freizügigkeit bei Arbeitnehmern eine ,Notbremse ' eingebaut. Diese wurde nun dahingehend verstärkt, dass der Europäische Rat in solchen Fällen auch gar nicht tätig werden muss. ${ }^{4}$

Das Vereinigte Königreich ,genießt' bereits derzeit viele opt-outs im Bereich der Justizund Innenpolitik und Schengen. Als direkte Folge der Zusammenführung der dritten mit der

2 Vgl. Erklärung Nr. 5 des Verfassungsvertrags. Der Ioannina-Mechanismus wurde selten angewandt: einmal, in besonderem Maße, bei einem Disput über portugiesische Fischereien.

3 Artikel 308 EG-Vertrag.

4 Artikel 42 EG-Vertrag. 
ersten Säule wird die Regierungskonferenz beauftragt, den Geltungsbereich des Protokolls hinsichtlich des Vereinigten Königreichs auf die Bereiche der justiziellen und polizeilichen Zusammenarbeit auszudehnen. ${ }^{5}$ Eine Bewertung der opt-outs des Vereinigten Königreichs im Schengenbereich wird ebenfalls erwartet. ${ }^{6}$ Jedoch, wenn das Vereinigte Königreich eine Blockadehaltung wählen wird, wird dies den Rest der Union antreiben, die Bestimmungen zur verstärkten Zusammenarbeit anzuwenden (mindestens neun Mitgliedstaaten müssen sich beteiligen), insbesondere im Hinblick auf Eurojust und die operative Polizeiarbeit.

Als Zugeständnis an Frankreich und die Niederlande wird ein Protokoll eingefügt, mit dem Ziel, Dienste von allgemeinem wirtschaftlichen Interesse vor den Unerbittlichkeiten des Gemeinsamen Marktes zu schützen. Es ist unklar, wie dies dazu beitragen wird, die Dinge zu klären, außer dass bekräftigt wird, dass nicht-wirtschaftliche Dienstleistungen von allgemeinem Interesse, wie öffentliche Gesundheit, in die alleinige Zuständigkeit der Mitgliedstaaten fallen. Ob es weise war, Protektionismus in den Rang des Primärrechts zu erheben, wird die Zukunft zeigen.

Als Zugeständnis an Frankreich wird der neue Artikel über die Ziele der Union dahingehend geändert, dass das Konzept des „freien und unverfälschten Wettbewerbs“ nicht mehr genannt werden wird. Es wird jedoch ein Protokoll hinzugefügt werden, damit die Kommission weiterhin sicherstellen kann, dass der „Wettbewerb vor Verfälschungen geschützt“ wird, und damit die Rechtsprechung, die die Wettbewerbspolitik als eines der zentralen Ziele der Union bestätigt, unberührt bleibt.

\section{Das Europäische Parlament als konstitutioneller Akteur}

Die größere Rolle des Europäischen Parlaments in der Regierungskonferenz ist ein Zeugnis für seine wachsende Glaubwürdigkeit als konstitutioneller Akteur. Durch die sehr aktive Beteiligung im Konvent (2002-03) ist das Parlament dafür mitverantwortlich, nun neue Vorschläge zu formulieren, um so viele Elemente des ursprünglichen Verfassungsvertrags wie möglich zu retten. Als Institution, die viel gewonnen hatte in den Verhandlungen über die Verfassung, dringt das Parlament auf eine zügige Beilegung der Streitigkeiten, die das Inkrafttreten verhindern.

Dennoch, wie alle anderen, die an diesen komplexen mehrstufigen und mulilateralen Verhandlungen beteiligt sind, fordern die Mitglieder des Europäischen Parlaments ihren Preis. Schlüsselelemente sind die Abschaffung der dritten Säule, die rechtsverbindliche Charta der Grundrechte, die neuen Bestimmungen zur Außenpolitik, die Rechtspersönlichkeit sowie, natürlich, die Ausweitung des Mitentscheidungsverfahrens. ${ }^{7}$ Durch das Ratifikationschaos hat das Europäische Parlament gelernt, dass es noch viel effektiver darin werden muss, die Medien, Parteien und Bevölkerungen vom europäischen Projekt zu überzeugen. Diesmal steht viel auf dem Spiel. Ein zweiter Fehlschlag wäre eine Katastrophe für das ganze Europa, innen- wie außenpolitisch.

Übersetzung aus dem Englischen von Tanja Leppik.

\footnotetext{
Protokoll Nr. 4, Amsterdamer Vertrag. Protokoll Nr. 3, Amsterdamer Vertrag. Europäisches Parlament: Entschließung zu der Roadmap für den EU-Verfassungsprozess, P6_TA-PROV (2007)0234 vom 7. Juni 2007.
} 